IZA DP No. 7896

Selfish Altruism, Fierce Cooperation and the Emergence of Cooperative Equilibria from Passing and Shooting

Nikos Askitas

January 2014 


\title{
Selfish Altruism, Fierce Cooperation and the Emergence of Cooperative Equilibria from Passing and Shooting
}

\author{
Nikos Askitas \\ IZA \\ Discussion Paper No. 7896 \\ January 2014 \\ IZA \\ P.O. Box 7240 \\ 53072 Bonn \\ Germany \\ Phone: +49-228-3894-0 \\ Fax: +49-228-3894-180 \\ E-mail: iza@iza.org
}

Any opinions expressed here are those of the author(s) and not those of IZA. Research published in this series may include views on policy, but the institute itself takes no institutional policy positions. The IZA research network is committed to the IZA Guiding Principles of Research Integrity.

The Institute for the Study of Labor (IZA) in Bonn is a local and virtual international research center and a place of communication between science, politics and business. IZA is an independent nonprofit organization supported by Deutsche Post Foundation. The center is associated with the University of Bonn and offers a stimulating research environment through its international network, workshops and conferences, data service, project support, research visits and doctoral program. IZA engages in (i) original and internationally competitive research in all fields of labor economics, (ii) development of policy concepts, and (iii) dissemination of research results and concepts to the interested public.

IZA Discussion Papers often represent preliminary work and are circulated to encourage discussion. Citation of such a paper should account for its provisional character. A revised version may be available directly from the author. 


\section{ABSTRACT}

\section{Selfish Altruism, Fierce Cooperation and the Emergence of Cooperative Equilibria from Passing and Shooting ${ }^{*}$}

There is continuing debate about what explains cooperation and self-sacrifice in nature and in particular in humans. This paper suggests a new way to think about this famous problem. I argue that, for an evolutionary biologist as well as a quantitative social scientist, the triangle of two players in the presence of a predator (passing and shooting in 2-on-1 situations) is a fundamental conceptual building-block for understanding these phenomena. I show how, in the presence of a predator, cooperative equilibria rationally emerge among entirely selfish agents. If we examine the dynamics of such a model, and bias the lead player (ball possessor with pass/shoot i.e. cooperate/defect dilemma) in the selfish direction by only an infinitesimal amount, then, remarkably, the trajectories of the new system move towards a cooperative equilibrium. I argue that "predators" are common in the biological jungle but also in everyday human settings. Intuitively, this paper builds on the simple idea - a familiar one to a biologist observing the natural world but perhaps less so to social scientists - that everybody has enemies. As a technical contribution, I solve these models analytically in the unbiased case and numerically by an $O\left(h^{5}\right)$ approximation with the Runge-Kutta method.

JEL Classification: $\quad$ C71, C73, C57, D87

Keywords: evolutionary game theory, fitness, altruism, evolution of cooperation, decoy, Nash equlibrium, repeated matching-pennies game, predator, emergence, autonomous ODE, classical Runge-Kutta method

Corresponding author:

Nikos Askitas

IZA

P.O. Box 7240

53072 Bonn

Germany

E-mail: nikos@iza.org

\footnotetext{
* Earlier versions of this paper were presented at the IZA brown bag seminar on two occasions (February 2012 and January 2013).
} 
IZA Discussion Paper No. 7896

January 2014

\section{NON-TECHNICAL SUMMARY}

What can soccer teach us about life? If you are like millions of other people the answer is: everything. Soccer is among many other things also about luck and ability, randomness and order, talent and hard work, homeostatic variations, schadenfreude and envy, physical and mental exhaustion, saturation, diminishing greed, instinct and intellect, sweat, blood, money and poverty, expensive cars (conspicuous consumption) and pretty women, cooperation and defection, fairness and cheating, rules and the breaking of rules, individual abilities and tactical systems, camaraderie (group identity), loyalty and betrayal, bragging rights, rankings, institutions, gambling, data.

To motivate our paper we need to go back to January 292011 when Bayern Munich had just beaten Werder Bremen 3-1 in Bremen. The highlight of the game was a confrontation between teammates Arjen Robben und Thomas Müller. Robben had just returned from an injury and his shooting to passing ratio was apparently excessively on the selfish side for Müller's taste and Müller let him know about it by wildly gesticulating in Robben's direction. Robben who felt this was a sign of luck of "respect" grabbed Müller by the neck and let him know how he felt about it. Towards the end of the same year, in an interview with chrismon.de Philipp Lahm, a teammate of Müller and Robben, when asked whether the myth of eleven friends were true answered: "No. Viewed realistically a team consists of two-dozen competitors. Of course they all have nonetheless success as a common goal. But I would not advise any professional player to confide intimate things in the team, for example about a phase of weakness that he is going though." When asked why he would not advise so he answered: "One confides to friends, not to teammates, who are just lurking around for a sign of weakness on one's part."

So soccer is about cooperation and defection, about "being there" hoping for a pass and being used as a decoy instead, about guarding the player with ball possession or the spotter positioning for a pass. In this paper we model two-on-one situations i.e. two strikers against one defender by identifying their dilemmas. The ball possessor's dilemma is whether to shoot or to pass while the player who positions for a pass (the decoy) is faced with a dilemma to decoy or to spite his teammate. The defensive player's predicament is to compute how to allocate his defensive energies: guard ball possession or guard the pass?

We study the dynamics of three players playing a three way game of 2-on-1. We assume that the players are evolutionary in the sense that once they compute their success rate they modify it so that they do better. So one striker adapts his shoot-to-pass ratio while his teammate varies his decoy-tospite ratio and the defensive player his guard-left-to-guard-right ratio.

We show that if we bias the shooter so that he depicts an even infinitesimal reluctance to reduce his shooting (but he nonetheless adapts evolutionarily) then surprisingly we are lead to a cooperative situation in which the striker shares the ball fairly with his teammate/spotter who is in turn completely loyal to decoying for him. We then say that cooperation and altruism are emergent phenomena not despite but because of selfishness and hence we speak of "selfish altruism" and "fierce cooperation".

We think that the triangle of two players in the presence of a predator ought to be a fundamental block for the understanding of the emergence of cooperation and altruism in quantitative social science and evolutionary biology alike. In natural experiments fish like guppies and sticklebacks perform what is known as "predator inspection" i.e. assess the danger when predator fish are around. When accompanied by a teammate (2-on-1) they are more confident in approaching the danger than when not.

While behavioral economics tries to understand the workings of human cooperation and altruism, in evolutionary biology a fundamental question is to explain the antinomy between the selfish gene and the prevalence of cooperation and altruism. While Darwinian evolution with its natural selection is based on unmitigated selfish competition it nonetheless selects cooperative, self-sacrificing phenotypes. We show that the predator explains this antinomy both in the jungle as well as society the economy and the formation of nations. Our proposition is based on what biologists know well and social scientists don't always remember but is ubiquitous: everybody has enemies. 


\section{INTRODUCTION}

Cooperation and altruism play a fundamental role in the formation of complex life forms out of simpler ones. This statement is true in a "scale invariant manner" from the protozoan ooze of the depths of time to the formation of firms, economies and nations ${ }^{1}$. The abundance of instances of cooperation and altruism, on the other hand, contradicts selfish competition, the central axiom of evolution. This antinomy makes the understanding of these phenomena important research subjects in both economics and evolutionary theoretical biology and reminds us of the proximity of the two sciences much of whose overlap occurs within game theory ${ }^{2}$.

Inspired by passing and shooting in two-on-one situations in soccer/basketball and its similarity to predator inspection in certain types of fish (Dugatkin (1988) and Milinski (1987)) we show that, in the presence of a predator, cooperative equilibria are emergent phenomena not despite selfishness but because of it. We do so by studying the dynamics of three evolutionary agents playing a matching-pennies game which involves a goalie/predator $Z$ with a defensive allocation dilemma and a striker pair $X, Y$ with shoot-pass and decoy-spite dilemmas. For the sports fans among the readers the situation appears again and again in team sports in the form of two-on-one fast breaks, "pick and roll", or two strikers in the finishing stages of a play where the striker with the ball must decide whether to shoot or to pass. We model this situation in the form of a stage game and studying it in repetition results in a truncated autonomous system of ODEs describing the ensuing dynamics. We show that if we bias player $X$ (the ball possessor) in the selfish direction by infinitesimally perturbing his ODE then, surprisingly, the trajectories of this new system of ODEs (solved numerically by an $\mathcal{O}\left(h^{5}\right)$ approximation using the classical Runge-Kutta method) flow towards a cooperative equilibrium where $X$ passes half the time, $Y$ decoys all the time and $Z$ defends by flipping a coin.

\footnotetext{
${ }^{1}$ See Bowles and Gintis (2011) for a wonderful account of a good deal of this story and an excellent starting point into a good portion of the existing literature.

${ }^{2}$ It is an extremely interesting experience for example to read Fontaine (2008) and trace how biological ideas on altruism mutate and reincarnate themselves in the economic literature.
} 
We are well aware that our setting is a departure from the "orthodox paradigm" used to study cooperation and altruism but we think that it is nonetheless valuable in that it adds a novel mechanism which explains why and how "cooperation, altruism, and self-sacrifice" rise and fall with "the great heartbeat of nature" (Nowak and Highfield (2011)).

In a game of two-on-one i.e. two strikers against one defender/goalie, the player with ball possession may choose to shoot (defect) or pass (cooperate or be altruistic) while his teammate may choose to decoy (i.e. make himself available for a pass i.e. cooperate) or defect (this may range from just resigning or holding back to sabotaging). The defender's predicament is the optimal allocation of defensive energy (the allocation ranges from defending the ball possession exclusively to defending the pass exclusively) while the two players are completely selfish and in fact want to outscore each other as the loser will perish. Complexity ensues in the repeated setting. The ball possessor, utilising the distraction of the defender by the decoy, will rack up goals and establish himself as the primary source of danger for the defender, by being completely selfish (never passes). He thus triggers two processes. The defender may increase his vigilance against him since he does not have to worry about the decoy (no passes occur) and at the same time the decoy may moderate his devotion and cease to decoy. Both of these new developments will make scoring increasingly hard for the ball possessor who eventually will be better off passing than shooting. As the ball possessor starts passing the defender has his entire defensive energy focused on ball possession and he is hence exposed to scoring from the decoy. The latter then reaps the benefits of the circumstance. It will not take long for the defender to revise his defence allocation and pay more attention to the decoy who meanwhile has become the prime source of danger. This will eventually make it worthwhile again for the ball possessor to shoot the ball to his own benefit. The decoy will tolerate the increased shooting for a while before he revises his devotion again. Eventually the defender will refocus back on ball possession and the cycle will repeat. These are the fundamental forces at play in a two-on-one setting. Both players are purely selfish but in a way indirectly kind to each other 
as they adopt their behaviour to the payoff volatility caused by the defender who, in his own turn, adjusts his defensive allocation according to the behaviour of the offensive players. It is this example which will inspire the rest of the discussion in this paper.

The evolutionary origin of the two-on-one situation is life in the presence of a predator. Anyone who ever observed a lion zig-zag-chasing two or more antelopes knows the value of not being alone in the visual field of a predator i.e. the value of having a decoy. While the predator zig-zags to "compute" the slowest prey, all the antelopes need to do is outrun their slowest companion. The same principle is at work in the various flavours of the school yard game of tagging: if you are "it" (i.e. the predator) you zig-zag-chase to tag the slowest child and if you are on the run you try to hide in numbers. An example of a more economic setting for the game of tagging is when company downsizings occur: the key to keeping your job is to simply outperform your colleagues and competitors but you need to have them around and be able to do so in the first place so you support them when you can. The presence of a predator provides us with the unique setting where the fact that life is packaged in many different, small and spatially distributed enclosures (and not in one big monolithic blob) unfolds its comparative advantage. In economic terms the predator may also be a regulator of some kind.

Cooperation is typically studied by means of two-player games with two-pure strategies for each player and certain conditions imposed on their payoff matrix. On the one hand, these conditions make, cooperating with a cooperator more profitable than defecting against a defector (this has an obvious evolutionary significance: populations where intra-cooperator encounters are common are doing better than those where defector encounters are more common) and on the other hand they give defection a conditional premium over cooperation (temptation vs sucker). In the economists' parlance these conditions create an opportunity cost of cooperating (the competing option being to defect) for any fixed strategy of the opponent. Formally if $T$ is the pay-off of a defector against a cooperator (the Tempation), $R$ is the payoff of cooperators playing against each other (the Reward), $P$ is the (Punishment payoff) of two defectors playing 
each other and $S$ is the (Sucker) payoff of a cooperator playing a defector then a game is called a cooperation game if $T>R>P>S$. In the repeated setting one imposes that $2 R>T+S$ to prevent players from averaging Temptation and Sucker rewards. A special case of such a game is prisoner's dilemma whose non-repeating version reaches its Nash equilibrium when both players defect because, due to the aforementioned conditions, this is how they maximise payoffs regardless of whether the opponent is a cooperator or a defector. The iterated prisoner's dilemma is the "orthodox paradigm for the evolution of cooperation among selfish individuals" (Nowak and Sigmund (1992)). An altruist in the literature is, like its vernacular counterpart, a player who foregoes some portion of his payoff or fitness to benefit others and all attempts to explain altruism in evolutionary terms use "models designed to take the altruism out of altruism" (Trivers (1971)) in other words to convert it to "as-if altruism" (Fontaine (2008)). A typical result is that altruism is non-altruistic i.e. it is (ultimately) beneficial to the self and hence evolutionarily preferred whenever the ratio of the cost of the benefactor to the benefit of the recipient of his altruism exceeds some threshold involving quantities such as the degree of relatedness between them.

A surprisingly common simplifying assumption across the two-player literature is that the payoffs of the stage game remain constant across encounters. Life on the other hand is often more complex than that and the payoffs of a pure strategy may depend on additional factors, other than the opponent's pure strategy, such as ambient environmental conditions (replicator dynamics often account for that - Pagen and Nowak (2002)) or the players' various biological "homeostatics". Homeostasis is called "diminishing returns" in economics: recent winnings devaluate a future winning increment causing an agent to put less vehemence into winning an additional unit. Similarly a totally selfish player who always shoots rather than passes suffers a deterioration of his scoring ability because the goalie figures him out. A beautiful neurological proof of the workings of these homeostastics in our context is the paper of Takahashi et al. (2009). Unfavourable comparisons (envy) in "domains of self-relevance" are shown to activate 
the anterior cingulate cortex, where pain is also known to take place while favourable ones activate the ventral striatum where rewards are processed. This nicely demonstrates our idea. Envy is, like pain, a self-preservation alarm prompting us to compete while schadenfreude is the reward we feel when we outcompete our competitor which causes us to diminish our vigilance. The long term effect of these homeostatics is what our paper attempts to highlight and perhaps explain: while outcompeting our adversaries/teammates is of evolutionary significance we are well advised to not completely eliminate them (hence to perhaps be altruistic or at least "not kick a man when he is down") because in the presence of a predator it is nice to have them around. While such things as fatigue or saturation are prime examples of such systemic homeostatics nothing explains their origin as well as a predator. A predator is the primordial generator of payoff variation in a repeated two-player stage-game and in fact explains well why homeostatics driven by envy and schadenfreude evolved in the first place. The reader will also indulge our suggestion based simply on intuition that a predator must have played a significant role in the formation of groups, group identity but also on human "gregariousness" (Hamilton (1971)) and "fashion" (Clark and Oswald (1998)).

The mechanisms currently known to partially explain how evolution favours cooperation (and to which we aspire to add our predator driven approach) are Haldane and Hamilton's "kin selection" (Hamilton (1963)), direct reciprocity in the repeated setting (Hamilton (1963)), a mechanism important in human behaviour (Falk and Fischbacher (2006)) and with many subtelties (Falk et al. (2005), Zizzo and Oswald (2009)), indirect reciprocity (players do not repeat-encounter directly but indirectly i.e. they play new players but carry along the aggregation of past encounters in the form of a reputation), group selection (in which groups of cooperators are shown to do better than groups of defectors), punishment, signalling ("green beard" models) etc. These mechanisms are important because they explain how evolution which works with mutation and natural selection nonetheless possesses the "remarkable ability to generate cooperation in a competitive world"( Nowak (2006)). 
The rest of the paper is organised as follows. We define the stage game in Section 2 motivated by the two-one-game above, compute its Nash equilibria and finish by writing down an autonomous (truncated) system of ODEs which describe the repeated game in the continuous setting. In Section 3 we solve the system of ODEs analytically and define a new system by perturbing the original system. The perturbation amounts to biassing the ball possessor in the selfish direction. We solve this new system numerically by means of the classical Runge-Kutta method. We close with a discussion of the consequences of our main result.

\section{THE STAGE GAME: MATCHING PENNIES}

We now define our 3-player stage game ${ }^{3}$. A goalie $Z$ is faced with the predicament of defending a striker duo $X$ (ball possessor) and $Y$ (a decoy or spotter). Striker $X$ 's predicament is to either shoot the ball (this is the selfish behaviour and we say that $X$ plays 0 ) or pass it to $Y$ (this is the cooperative or altruistic behaviour and we say that $X$ plays 1). The options of $Y$ are to spot $X$ in the attack (i.e. to decoy/cooperate and we say that $Y$ plays 0 in this case) or hold back (to spite/defect and we say that $Y$ plays 1). The goalkeeper's predicament is how to divide his defensive energies. Figure 1 depicts in "X's and O's notation" our passing stage game. We assume that once $Z$ guesses correctly he is fully successful at defending his goalpost

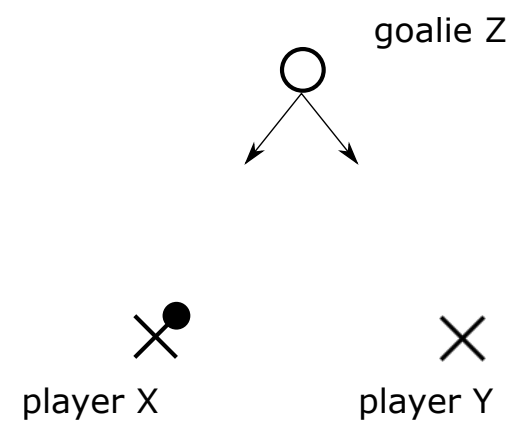

FiguRE 1.- Two strikers against one goalie in "Xs and Os notation"

and hence our game is in the "matching pennies" family. When $Z$ defends $X$ we say that $Z$

\footnotetext{
${ }^{3}$ Economics and Biology literature seem to have conspicuously kept a certain distance to three or more player games perhaps because they are known to be PPAD and hence hard (Daskalakis and Papadimitriou (2005)).
} 
plays 0 and when he defends $Y$ we say that he plays 1 . Let $x_{i}, z_{i}$ be the probabilityies of $X, Z$ to play $i$ and let $y$ be the probability of $Y$ to decoy (i.e. to play 0 ). We summarise all this in the payoff matrix (2.1) below ${ }^{4}$. The payoffs are arranged in a triangle (inherited from Figure 1 ) in each of the four cells containing the options so that at the top is the payoff of $Z$ and the bottom left is the payoff of $X$ and bottom right is the payoff of $Y$. The asymmetry between players $X$ and $Y$ i.e. between ball possessor and decoy is reminiscent of the Stackelberg Model of Duopoly (see for example Gibbons (1992)).

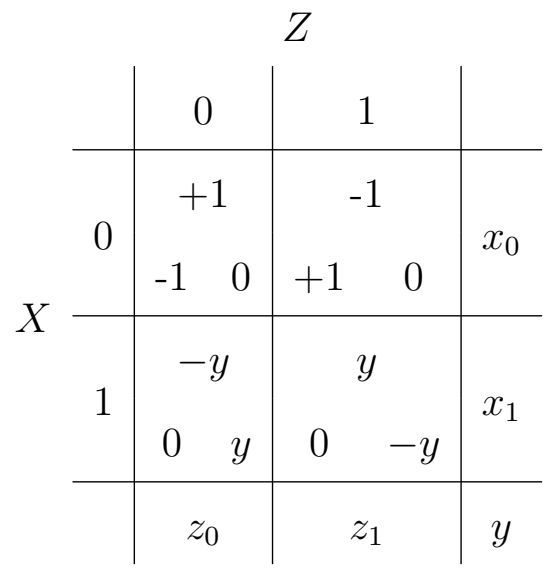

We can now write the expected payoffs $E U_{w}$ of each player $w \in\{X, Y, Z\}$ as a function of their mixed strategies. We simplify notations as follows. Since $x_{1}=1-x_{0}$ and $z_{1}=1-z_{0}$ we set $x=x_{0}$ and $z=z_{0}$ so that $x, y, z$ are the mixed strategies of $X, Y, Z$. In other words $x$ is the probability that $X$ plays 0 i.e. that $X$ is selfish and $y$ is the probability that $Y$ plays 0 i.e. that $Y$ decoys (is cooperative) and $z$ is the probability that $Z$ plays 0 i.e. that $Z$ defends against $X$ :

\footnotetext{
${ }^{4}$ For the convenience of the reader the mnemonic rule for assigning values is as follows. We fix the convention of thinking of $X$ as player 0 and $Y$ as player 1 and everything else follows from that. That means when $X$ plays 0 (resp. 1) he plays in favour of player 0 (resp. 1) i.e. he shoots (resp. passes). Similarly for $Y$ to play 0 (resp. 1) is to favour $X$ (resp. $Y$ ) i.e. to spite (resp. decoy). Finally $Z$ plays 0 when choosing to defend against the shot i.e. player 0 .
} 
$(2.2 \mathrm{a})$

$$
\begin{aligned}
(2.2 \mathrm{a}) \quad E U_{X} & =-z_{0} x_{0}+z_{1} x_{0} \\
& =x(1-2 z), \\
(2.2 \mathrm{~b}) \quad E U_{Y} & =z_{0} x_{1} y-z_{1} x_{1} y \\
& =y(1-x)(2 z-1), \\
(2.2 \mathrm{c}) \quad E U_{Z} & =z_{0} x_{0}-z_{1} x_{0}-y z_{0} x_{1}+y z_{1} x_{1} \\
& =(2 z-1)[x(y+1)-y]
\end{aligned}
$$

By setting the partial derivatives to zero as in (2.3) below and solving the equations we easily see that the set of Nash equilibria of the stage game is the curve $\{(x, y, z): x=y /(y+1) \wedge z=$ $1 / 2\}$ in the unit cube $[0,1] \times[0,1] \times[0,1]$ of mixed strategies. Notice that the hyperplane $z=1 / 2$ is the indifference locus of $X$ and $Y$ and the hypersurface $x=y /(y+1)$ is the indifference set of $Z$.

$$
\begin{aligned}
& \text { (2.3a) } \frac{\partial E U_{X}}{\partial x}=1-2 z=0, \\
& \text { (2.3b) } \frac{\partial E U_{Y}}{\partial y}=(1-x)(2 z-1)=0 \\
& \text { (2.3c) } \frac{\partial E U_{Z}}{\partial z}=2[x(y+1)-y]=0
\end{aligned}
$$

We now assume that $X, Y$ and $Z$ are evolutionary agents which play mixed strategies $x=$ $x(t), y=y(t)$ and $z=z(t)$ at time $t$ and which follow gradients of increasing returns. In other words that the system of autonomous ODEs (2.4) describes the dynamics of such a repeated game.

$$
\begin{aligned}
& \frac{d x}{d t}=1-2 z(t) \\
& \frac{d y}{d t}=(2 z(t)-1)(1-x(t)) \\
& \frac{d z}{d t}=2[x(t)(y(t)+1)-y(t)]
\end{aligned}
$$

Notice that these equations are related to the well known Lotka-Volterra replicator equations 
(see Pagen and Nowak (2002) for a unifying treatment of evolutionary dynamics). In fact if we multiply their right hand side by $x(1-x), y(1-y)$ and $z(1-z)$ respectively then we get the Lotka-Volterra replicator equations. In that case for $Y$ (for example) to be playing 0 (in the eyes of $X$ and $Z$ ) with probability $y$ means simply that $y$ is the share of the population of $Y$ s that play 0 (not necessarily the global share but the share among those $Y$ s that $X$ and $Z$ encounter and saved in their -however long- memory on which they base their "revision protocol"). Similarly for $X$ and $Z$ in the eyes of $Z, Y$ and $X, Y$ respectively. Our mixed strategies then are what an individual will experience moving in the crowd and randomly playing pass/shooting games in triangles. Our equations are the "revision protocols" (Sandholm (2006)), in other words, of agents with limited perception of the world, hence our language that what we are showing is that cooperation and altruism are emergent phenomena. In the next section we will study (a perturbation of) this system of ODEs (2.4) and write down the trajectories of the resulting phase space.

\section{SOLVING THE DYNAMICS OF SHOOTING AND PASSING}

We perturb the system of ODEs (2.4) by adjusting the equation (2.4a) so that $X$ now displays a selfish bias. We do that by adding to the right hand side of equation (2.4a) a non negative infinitesimal $\epsilon$ which is zero in a small neighbourhood of $z=1 / 2$. We glue the two parts by a "smooth step" function $\epsilon$ which respects $X$ 's indifference at $z=1 / 2$. More precisely for constants $\alpha, \beta, \delta \geq 0$, and $u=\frac{z-p+\delta}{2 \delta}, v=\frac{z-q+\delta}{2 \delta}$ let:

$$
\epsilon(z)= \begin{cases}\alpha & \text { if } z<p-\delta \\ \alpha\left(2 u^{3}-3 u^{2}+1\right) & \text { if }|z-p| \leq \delta \\ 0 & \text { if } p+\delta<z<q+\delta \\ \beta\left(-2 v^{3}+3 v^{2}\right) & \text { if }|z-q| \leq \delta \\ \beta & \text { if } z>q+\delta\end{cases}
$$


The smooth step function looks as in Figure 2 below.

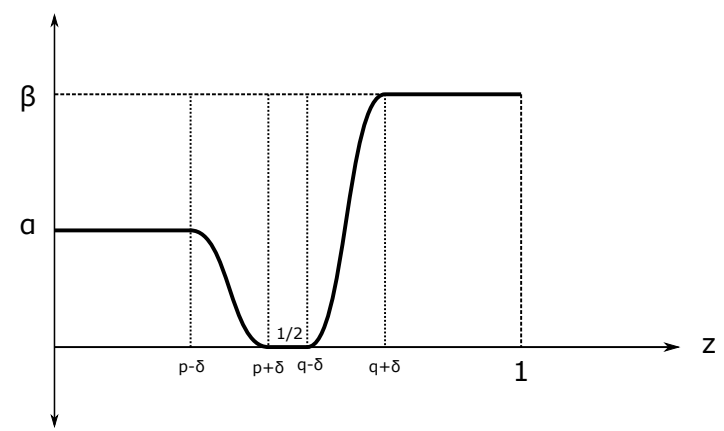

FiguRE 2.- The smooth step function $\epsilon$

The important thing about $\epsilon$ is that it is differentiable, non-negative and zero in a neighbourhood of $z=1 / 2$. The actual values are unimportant. We can think of all parameters $\alpha, \beta, \delta$ as infinitesimal quantities. We say that this bias of $X$ is in the selfish direction because the new equation (3.2a ) says that while $X$ follows the gradient of increasing returns he shows an $\epsilon$ bias in the direction of $x=1$ (the shooting direction and hence semantically the selfish one). Since we add $\epsilon$ to the derivative $(d x / d t)$ we insinuate that $X$ displays an infinitesimal reluctance to moderate his shooting which is not an unnatural condition to hypothesize. The perturbed version of the the system of ODE's (2.4) is then as follows:

$$
\begin{aligned}
& \frac{d x}{d t}=1-2 z(t)+\epsilon \\
& \frac{d y}{d t}=(2 z(t)-1)(1-x(t)) \\
& \frac{d z}{d t}=2[x(t)(y(t)+1)-y(t)]
\end{aligned}
$$

Using elementary methods equations (3.2a) and (3.2b) allow us to write $y$ as a function of $x$ (3.3a) and plugging that into equation (3.2c) we can write an implicit relationship of $z$ and $x$ (3.3b): 


$$
\begin{aligned}
y= & \frac{1}{2}(1-x)^{2}+\int^{t}(1-x(s)) \epsilon(s) \mathrm{d} s+c, c=\text { const. } \\
(1-2 z+\epsilon)^{2}= & -(1-x)^{4}-4(\lambda+1)(1-x)^{2}+8(1-x) \\
& +\int^{t}\left[4(1-x)^{3} \epsilon+2(1-2 z+\epsilon) \epsilon^{\prime}\right] \mathrm{d} s+d, d=\text { const. }
\end{aligned}
$$

Equations (3.3a) and (3.3b) completely describe all trajectories in the phase space which stay in the interior of the unit cube of mixed strategies. On the boundary of the unit cube where at least one of the players plays a pure strategy the reduction of the system (3.2) to a 2-variable system describes the dynamics until we enter the interior of the unit cube again. Notice that for $\alpha=\beta=0$ (i.e. $\epsilon=0$ ) the trajectories of the phase space are concentric closed curves given by quartics projected onto the one parameter family of surfaces $y=\frac{1}{2}(1-x)^{2}+c$. The motion on these closed trajectories is clockwise viewed from the positive $y$-axis.

The general idea of the behaviour of this system of ODEs, when $\alpha \neq 0$ or $\beta \neq 0$, is that regardless of starting point $x=x(0), y=y(0), z=z(0)$ its trajectory will revolve around $x=y /(y+1) \wedge z=1 / 2$ with $y$ increasing in the long run. From equation (3.3a ) we see that since $\epsilon$ is almost everywhere constant $y$ and $x$ will follow half-parabolas flatter than $y=\frac{1}{2}(1-x)^{2}+c$ when $z<1 / 2$ and slightly shifted towards $y=0$ and steeper and slightly shifted towards $y=1$ when $z>1 / 2$ with total net effect that $y$ will increases. Figure 3 schematically displays this:

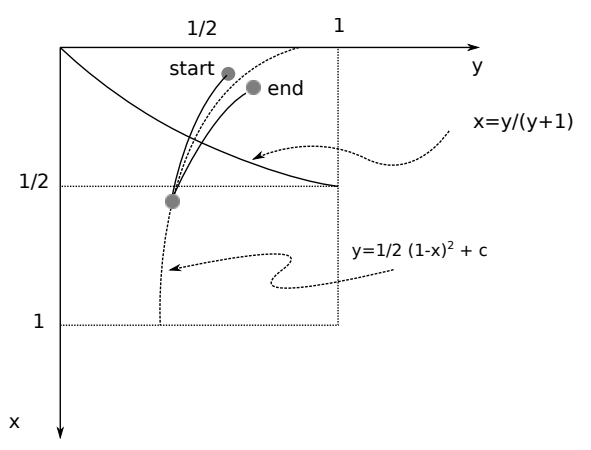

Figure 3.- $y$ increases in time when $\alpha \neq 0$ or $\beta \neq 0$ 
For $\alpha+\beta \neq 0$ the system has no analytic solutions (and a linearisation produces a Jacobian with zero eigenvalues) but using the Runge-Kutta method of numerical approximation of the solution (with local error in $O\left(h^{5}\right)$ ) we can demonstrate the behaviour of the system. In Figure 4 below we see how we reach the cooperative equilibrium when $\epsilon>0$ regardless of initial conditions. We orbit around the Nash equilibrium curve of the stage game just like in the non-perturbed case except now the orbit is not a closed curve. While we orbit around the Nash curve, $y$ steadily grows until it hits the wall $y=1$. At that point we truncate the system of three ODEs down to two because so long as $Y$ sees no reason to reduce $y$ we just need the dynamics of $X$ and $Z$. The reduced system of two ODEs keeps on alternating with the full system (as $y$ becomes equal to 1 again and again stays there for a while becomes somewhat smaller and back to 1 again). All the while $x$ and $z$ converge to $1 / 2$ :
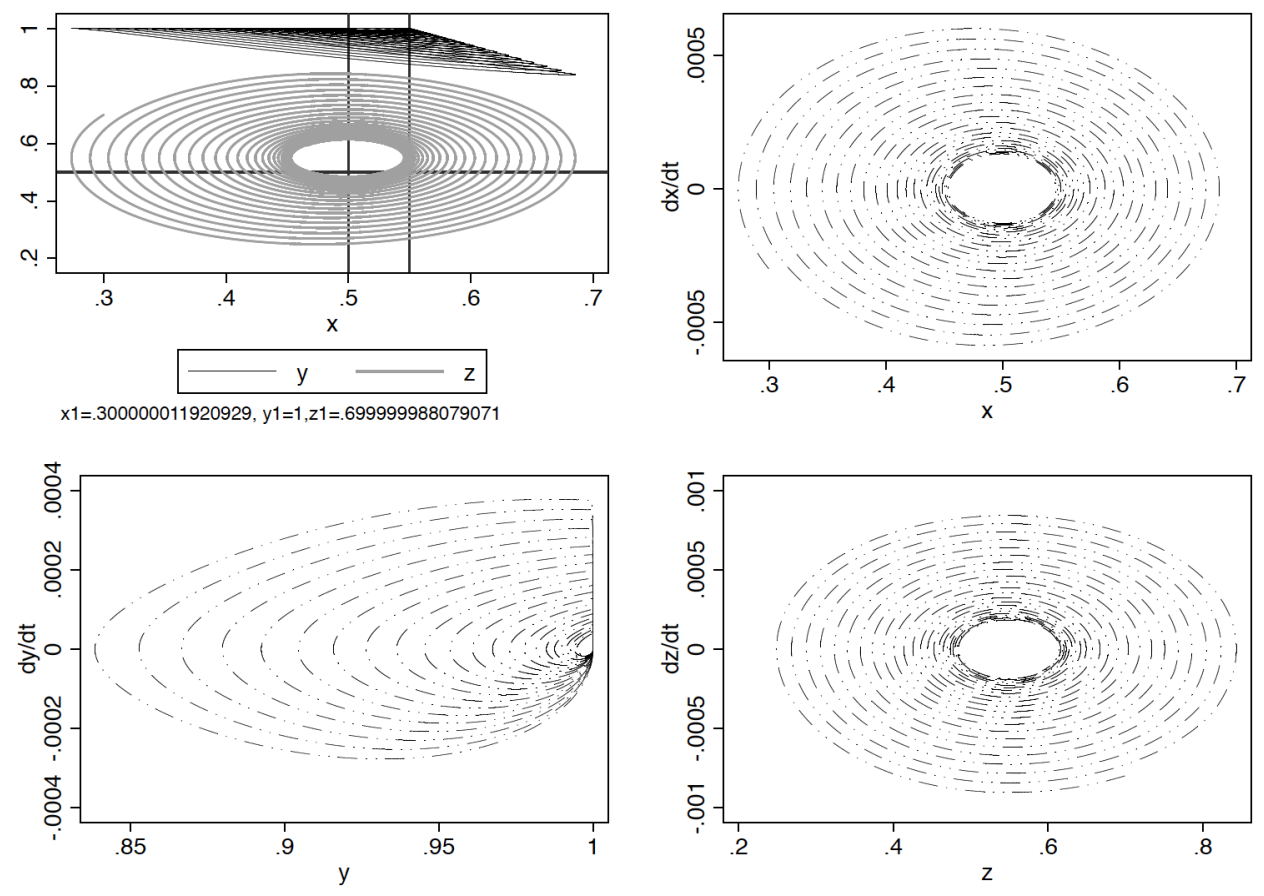

Figure 4.- Using the classical Runge Kutta method (p. 180 of Butcher (2008)) to solve the system of ODE 2.4 with $x(0)=.3, y(0)=1, z(0)=.7$ and $\alpha=.1, \beta=.1, \delta=.01$ and $h=.001$ 
Two remarks are in order here. First notice that our smoothness assumptions are not essential to our intended application and that hence our numerical approximation is not a weakness in our argumentation. Quite the opposite our computing algorithm shows that even weaker assumptions on the selfishness of $X$ will lead to the ODE converging to the same cooperative equilibrium. For example even if we just theorise very mild displays of hyper-selfish reluctance of $X$ to moderate his shooting we will still converge to the same sustainable cooperative equilibrium. The only thing that will change is the speed by which we approach it. Secondly notice that if we bias $X$ with $\epsilon<0$ i.e. the altruistic direction the system will converge to the unsustainable equilibrium $x=0$ ( $X$ always passes), $y=0$ ( $Y$ always defects) and $z=1 / 2$ (see Figure 5). This equilibrium is unsustainable because there is no chance of scoring for either $X$ or $Y$ and hence such species will not survive to tell us about it.
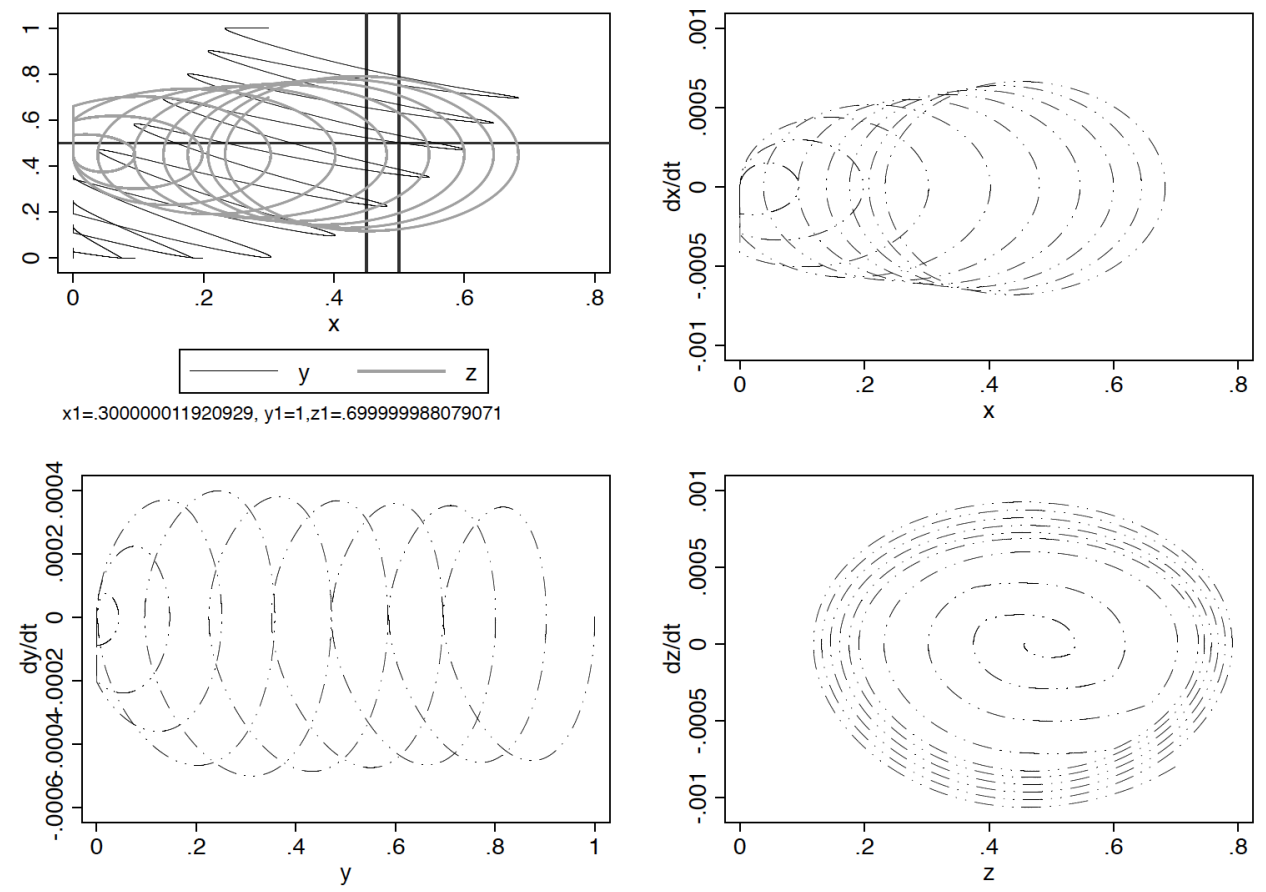

Figure 5.- The system of ODE 2.4 with $x(0)=.3, y(0)=1, z(0)=.7$ and $\alpha=-.1, \beta=$ $-.1, \delta=.01$ and $h=.001$ converging to an unsustainable equilibrium. 


\section{CONCLUDING REMARKS}

Our matching-pennies, 3-player game is on the border of where PPAD and hence computational hardness is believed to start and hence any situation in which life and its agents are trying to compute and reach a Nash equilibrium may have a hard time reaching it in general. Nonetheless we believe that the triangle of two players in the presence of a predator ${ }^{5}$ is one of the fundamental blocks for furthering our understanding of "cooperation, altruism and selfsacrifice". Two-on-one fast breaks in soccer give us a toy example to understand these issues in a new if somewhat unorthodox way. Our soccer application is that if you are a coach you want your strikers to be intelligent enough to pass but selfish enough to shoot rather than pass. The defender/predator will take care of the rest and before you know it they will tiki-taka their way to super-cooperative dominance. We have shown that whenever the lead player is biased "in the selfish direction" (i.e. displays an even infinitesimal preference towards shooting rather than passing - $\epsilon>0-)$ the long term equilibrium is a cooperative and sustainable one where the lead player shares the ball fairly, the decoy (teammate) is perfectly devoted to the lead player and the predator/goalie flips a fair coin to allocate his defensive energies. The oppositely signed bias $(\epsilon<0)$ leads to the other end of the stage game equilibrium line, where the lead player totally surrenders the ball to the decoy but the decoy is never there to receive it (ball possessor disappoints the decoy, decoy abandons ball possessor and ball possessor begs the decoy to come back in vain) and the predator flips a coin. That equilibrium is unsustainable and such triangles will perish as there is no chance of scoring. Purely statistically it is a lot more likely that the bias will be non-zero (a statement a mathematician would summarise by saying that "being biased is generic or transversal"). So, to recap, starting from randomly chosen initial states in the unit cube of mixed strategies, a non-biased system will orbit around the Nash

\footnotetext{
${ }^{5}$ Note that even prisoner's dilemma, the "orthodox paradigm" for the study of cooperation, involves a (silent) third player: the interrogator. Its role is negligible of course because of the symmetry in the game. Our game is one in which the interrogator may discriminate...
} 
equilibrium locus of the stage game in closed trajectories for ever, its aggregate phenotype oscillating between shooting and passing, decoying and defecting and guarding the ball or the pass while a negative bias will lead to unsustainable and hence perishable ends leaving us with only the positive bias ("in the selfish direction") which leads to cooperative and sustainable equilibria. We call the positive bias a selfish one because that is what the semantics would suggest i.e. because this is the direction where a shooter actively draws utility by shooting to score. It could be argued that such a bias is in the altruistic direction since the agent is not adapting fast enough when he ought to be doing so. Even so though one thing remains constant: it is still the only bias which leads to sustainable, and cooperative at that, equilibria. If we chose to call the positive bias an altruistic one then the negative bias would be a selfish one and lead to unsustainable triangles that perish. So our phenotypical conclusion is either that "selfish behaviours lead to cooperative equilibria" or that "altruism is evolutionarily fitter than selfishness"). In a world of perpetual oscillations between cooperation and defection semantics are bound to be blurry so in the presence of a predator (and we know that every life form has enemies) "selfish altruism" and "fierce cooperation" are far from contradictory pairs.

\section{ACKNOWLEDGEMENTS}

I was inspired by talks on biology and economics by Andrew Oswald whom I would like to also thank for discussions, comments and readings of earlier versions along with Kostas Tatsiramos and Lorens Imhof.

\section{REFERENCES}

Bowles, S. And H. Gintis (2011): A Cooperative Species, Princeton University Press.

Butcher, J. C. (2008): Numerical Methods for Ordinary Differential Equations, John Wiley \& Sons, Ltd.

Clark, A. E. AND A. J. Oswald (1998): "Comparison-concave utility and following behaviour in social and economic settings," Journal of Public Economics, 70, 133155.

Daskalakis, C. And C. H. Papadimitriou (2005): "Three-player games are hard," Electronic Colloquium on Computational Complexity, 139, 81-87. 
Dugatkin, L. (1988): "Do guppies play TIT FOR TAT during predator inspection visits ?" Behav. Ecol. and Sociobiol., 23, 395-399.

Falk, A., E. Fehr, and U. Fischbacher (2005): "DRIVING FORCES BEHIND INFOrMaL SANCTIONS," Econometrica, 73, 20172030.

FAlK, A. AND U. FischBacher (2006): "A theory of reciprocity," Games and Economic Behavior, 54, 293315.

Fontaine, P. (2008): "Altruism, history of the concept," in The New Palgrave Dictionary of Economics, ed. by S. N. Durlauf and L. E. Blume, Basingstoke: Palgrave Macmillan.

Gibbons, R. (1992): Game Theory for Applied Economists, Princeton University Press.

Hamilton, W. D. (1963): "The Evolution of Altruistic Behavior," The American Naturalist, 97, 354-356.

_ (1971): "Geometry for the Selfish and Herd," Journal of theoretical Biology, 31, 295-311.

MiLINSKI, M. (1987): "TIT FOR TAT in sticklebacks and the evolution of cooperation," Nature, 325, 433-435.

NowaK, M. A. (2006): "Five Rules for the Evolution of Cooperation," Science, 314, 1560-1563.

Nowak, M. A. And R. Highfield (2011): Super Cooperators - Altruism, Evolution, and why we need each other to succeed., Free Press.

NowaK, M. A. And K. Sigmund (1992): "Titr for tat in heterogeneous populations," Nature, 355, 250-253.

Pagen, K. M. and M. A. Nowak (2002): "Unifying Evolutionary Dynamics," J. theor. Biol., 219, 9398.

Sandholm, W. H. (2006): Population Games and Evolutionary Dynamics, MIT Press.

Takahashi, H., M. Kato, M. Matsuura, D. Mobbs, T. Suhara, and Y. Okubo (2009): "When Your Gain Is My Pain and Your Pain Is My Gain: Neural Correlates of Envy and Schadenfreude," Science, 323, 937-939.

Trivers, R. L. (1971): "The Evolution of Reciprocal Altruism," The Quarterly Review of Biology, 46, 35-57.

Zizzo, D. J. And A. J. Oswald (2009): "Are People Willing to Pay to Reduce Others' Incomes?" Annales d'conomie et de Statistique, 39-65. 